\title{
Agenesis of the Left Internal Carotid Artery in the Right Aortic Arch with Isolation of the Left Innominate Artery Associated with Ruptured Cerebral Aneurysm: Case Report
}

\author{
Kouji Yamasaki, ${ }^{1}$ Kiyotaka Yokogami, ${ }_{1}^{1}$ Hajime Ohta ${ }^{2}$, Shinji Yamashita, ${ }^{1}$ Takuma Kawasoe, \\ and Hideo Takeshima ${ }^{1}$
}

\begin{abstract}
As we occasionally encounter congenital blood vessel abnormalities, their variety, embryology, and associated clinical problems must be understood. We report a case of a 50 -year-old man with subarachnoid hemorrhage due to a ruptured cerebral aneurysm at the internal carotid-anterior choroidal artery bifurcation who manifested extremely rare congenital anomalies, i.e., a right aortic arch, isolated left innominate artery, agenesis of the left internal carotid artery, and dysplasia of the basilar artery to the posterior cerebral arteries. This complex anomaly, to the best of our knowledge, has not been previously reported.
\end{abstract}

Keywords: anomaly of the aortic arch and cerebral circulation, intracranial aneurysm

\section{Introduction}

We occasionally encounter patients with congenital blood vessel malformations such as a right aortic arch (RAA) or agenesis of the internal carotid artery (ICA). We report a patient with an extremely complex congenital blood vessel anomaly who suffered subarachnoid hemorrhage (SAH) due to a ruptured cerebral aneurysm. The anomalies included RAA with an isolated left innominate artery, agenesis of the left ICA, and dysplasia of the posterior circulation.

\section{Case Report}

A 50-year-old man in deep coma was admitted to our hospital. He had a history of untreated hypertension. His grandfather and father had suffered ruptured cerebral aneurysms, and his sister an unruptured cerebral aneurysm. Computed tomography (CT) revealed SAH and an intracerebral hematoma in the right temporal lobe (Fig. 1A). Three dimensional (3D) CT angiography showed an aneurysm at the IC-anterior choroidal artery bifurcation (Fig. 1B). It was clipped via the pterional trans-Sylvian approach. Postoperatively he manifested no neurological deficits.

He had an extremely rare congenital anomaly that involved the aortic arch and cerebral arteries. 3D-CT angiography and digital subtraction angiography (DSA) showed RAA with

${ }^{1}$ Department of Neurosurgery, University of Miyazaki, Miyazaki, Miyazaki

${ }^{2}$ Department of Neurosurgery, Miyakonojo Medical Association Hospital, Miyazaki, Miyazaki

Received: July 16, 2014; Accepted: August 27, 2014 isolation of the left innominate artery (Fig. 2) and agenesis of the left ICA (Fig. 3). Dysplasia of the posterior circulation (Fig. 4) was observed from the middle of the basilar artery to the left posterior cerebral artery (PCA). No arteriovenous shunt was seen. The left anterior cerebral artery (ACA) and middle cerebral artery (MCA) were supplied from the

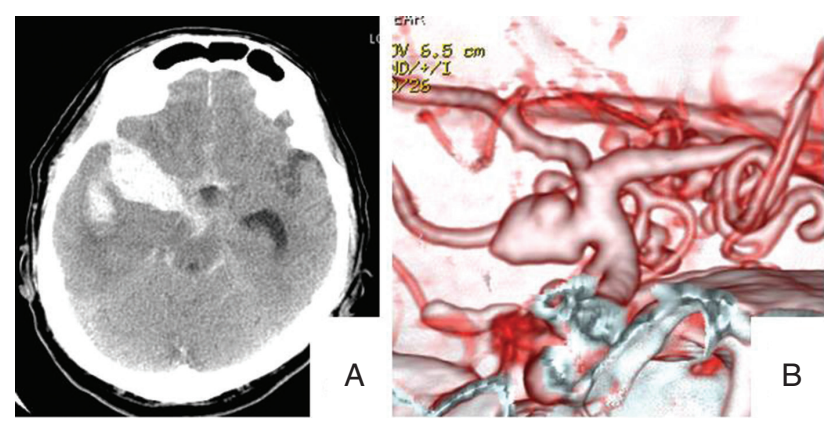

Fig. 1 A: Cranial CT showing SAH with intracerebral hematoma in the right temporal lobe. B: 3D CT angiography showing an aneurysm at the internal carotid-anterior choroidal artery bifurcation. 3D: three dimensional, CT: computed tomography, SAH: subarachnoid hemorrhage.
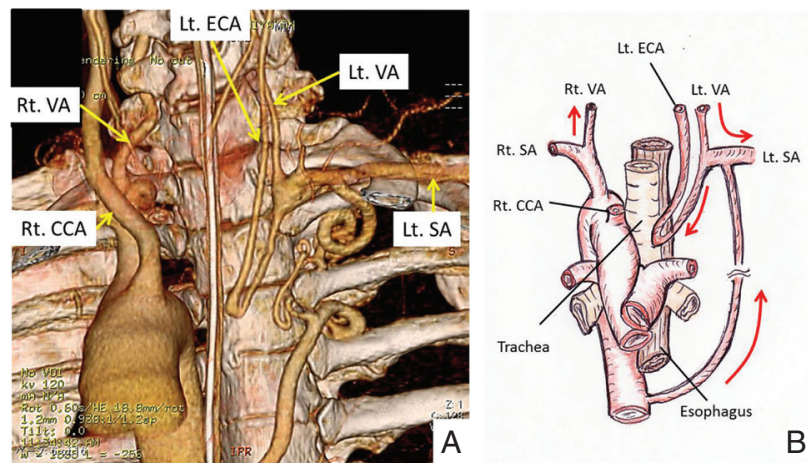

Fig. 2 A: 3D-CT angiography showing the right aortic arch with isolation of the left innominate artery. Left oblique view. B: Line drawing showing the right aortic arch with isolation of the left innominate artery. Red arrows indicate the direction of blood flow. Note blood steal from the left vertebral- into the left subclavian- and left external carotid artery. Collateral flow originating from the descending aorta to the left subclavian artery. CCA: common carotid artery, ECA: external carotid artery, VA: vertebral artery, SA: subclavian artery, Lt.: left, Rt.: right, 3D: three dimensional, CT: computed tomography. 

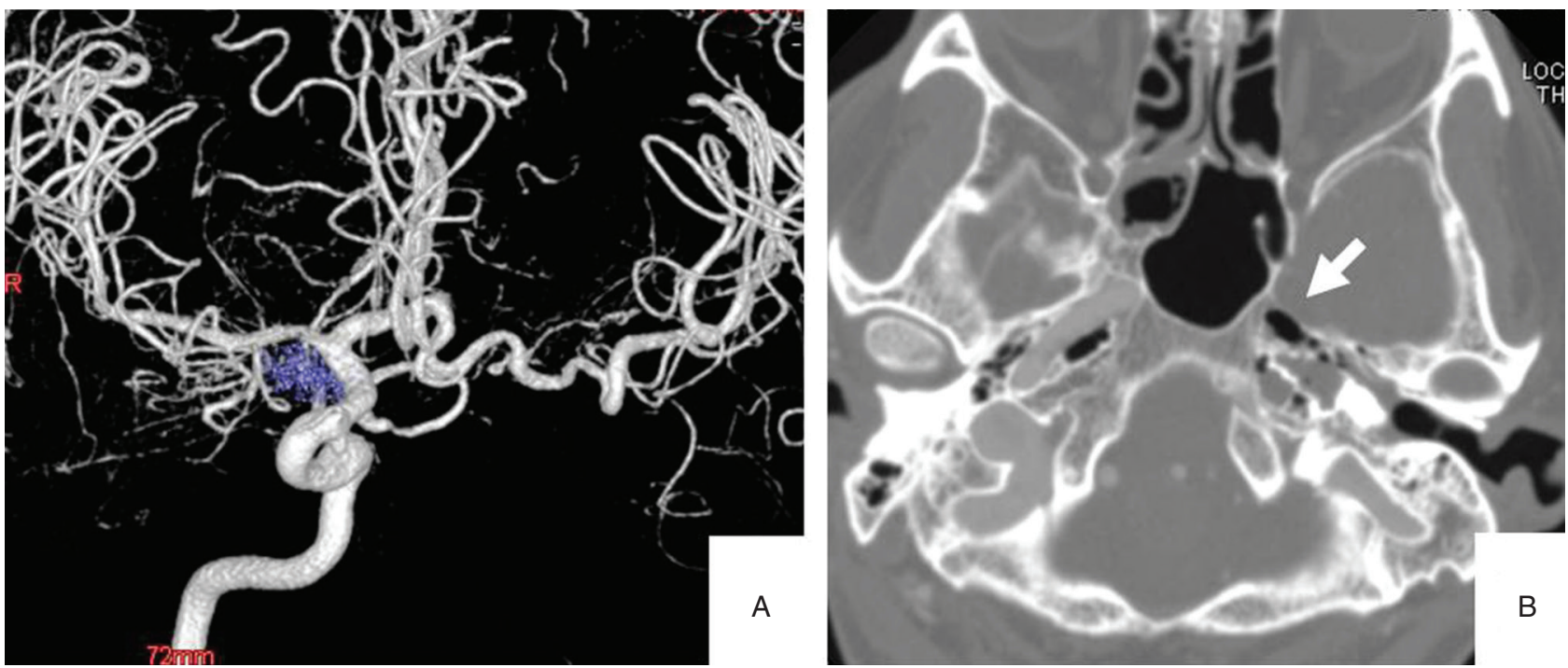

Fig. 3 Agenesis of the left ICA. A: Angiograph of the right ICA showing the collateral blood flow pathway. The left ACA and MCA were supplied across a patent AcomA. B: CT angiogram at the level of the petrous ICA showing absence of the carotid canal (axial image). ICA: internal carotid artery, ACA: anterior cerebral artery, MCA: middle cerebral artery, AcomA: anterior communicating artery, CT: computed tomography.
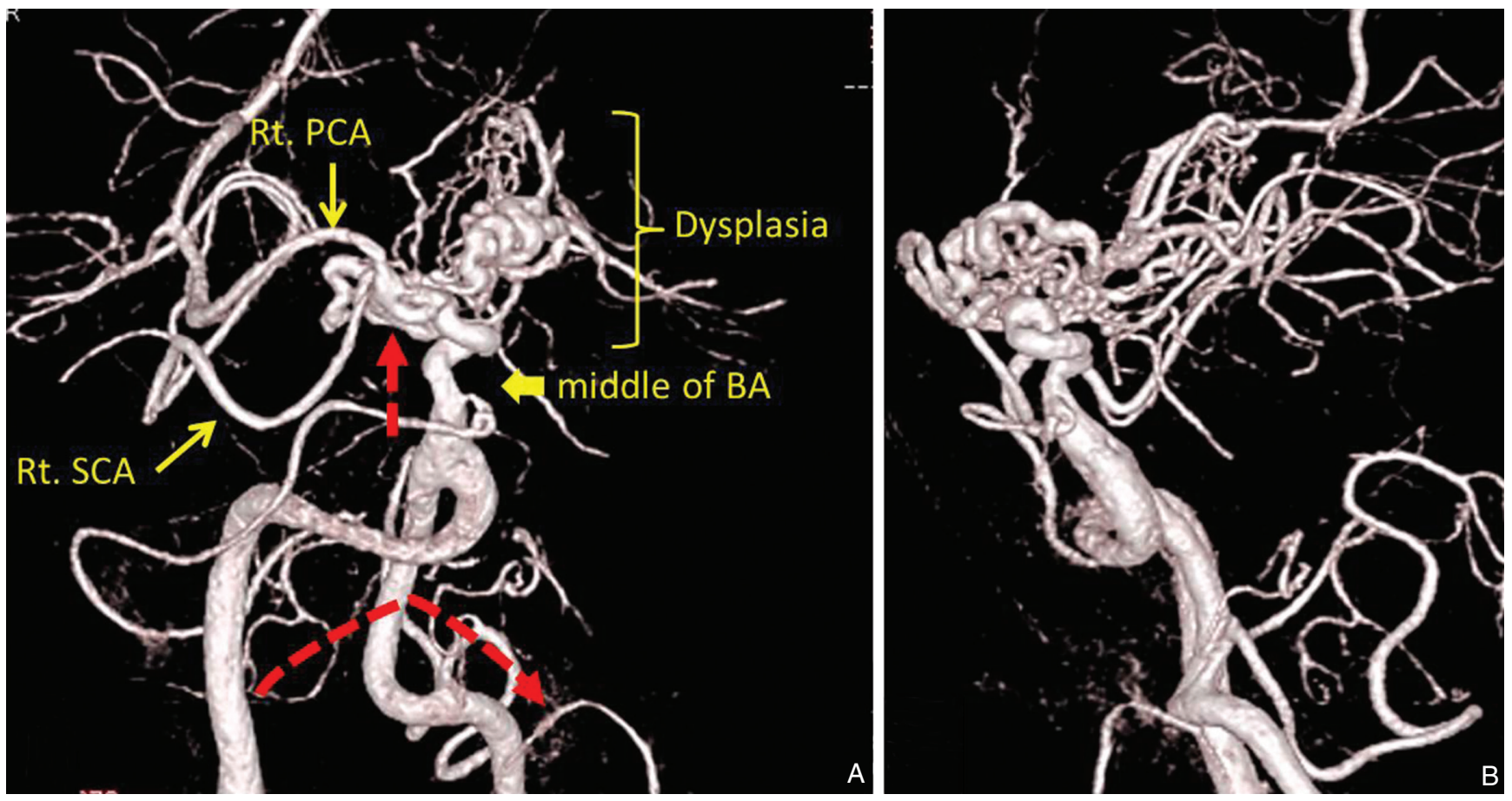

Fig. 4 A: 3D-angiograph of the right VA showing dysplasia of the posterior circulation on the left side from the middle portion of the basilar artery to the left posterior cerebral artery. A-P view. We cannot identify the left posterior cerebral artery and the left superior cerebellar artery exactly. Blood is flowing up in the right VA and down in the left VA. Some of the blood is distributed in the territory of the BA. Red arrows indicate the direction of blood flow. B: 3D-angiograph of the right VA showing dysplasia of the posterior circulation. Lateral view. PCA: posterior cerebral artery, SCA: superior cerebellar artery, BA: basilar artery, VA: vertebral artery, 3D: three dimensional, A-P: anterior-posterior, Rt.: right.

contra-lateral side via the anterior communicating artery (AcomA). The left subclavian- and left external carotid artery were supplied from the right vertebral artery (VA) via reverse flow in the left VA. There was collateral flow originating from the descending aorta into the left subclavianand left external cariotid artery.

\section{Discussion}

RAA with an isolated left innominate artery is an uncommon anomaly of the aortic arch. Stewart et al. ${ }^{1)}$ classified the RAA into three types: (1) RAA with mirror-image branching of the major arteries $(59.3 \%)$, (2) RAA with an aberrant left subclavian artery (39.5\%), and (3) RAA with an 
unconnected left subclavian artery $(0.8 \%)$. The RAA in our patient was not any of these types, although it is similar with (3) RAA with an unconnected left subclavian artery. It is unusual for patients to have this condition in adulthood without coexisting congenital cardiac anomalies; the association of a patent ductus arteriosus or other cardiac abnormalities, resulting in cardiovascular dysfunction, has been reported in neonates and in early life. ${ }^{2-4)}$ Agenesis of the ICA is also a rare congenital anomaly; it has been documented in less than $0.01 \%$ of the population. ${ }^{5)}$ Although a few instances of both aortic arch anomaly and agenesis of the ICA have been reported ${ }^{6)}$ ours is the first documentation of this type of complex anomaly and no patients with additional dysplasia of the posterior circulation have appeared in the literature.

Embryologically, RAA with an isolated left innominate artery is explained by regression of the developing aorta between the aortic sac and the left common carotid artery, degeneration of the eighth segment of the left dorsal aorta, and regression of the bilateral ductus arteriosus. ${ }^{3,4)}$ ICA agenesis involves the abnormal regression, involution, or atresia of the third aortic arches and dorsal aorta; the pathogenesis of dysplasia of the posterior circulation remains unknown. The basilar artery distal to the primitive trigeminal artery derives from the ipsilateral ICA and anastomoses with the vertebrobasilar system. We speculate that blood flow insufficiency plays a role in angiogenesis of the vertebrobasilar system. In the presence of agenesis of the ICA or RAA with an isolated left innominate artery, a coexisting anomaly of the vertebrobasilar system is extremely rare.

Among patients with agenesis of the ICA, the incidence of intracranial aneurysms is $24-34 \%$, and much higher than in the general population (2-4\%). Increased flow through collateral vessels and altered flow dynamics are cited as plausible explanations for this higher incidence. ${ }^{7-9)}$ On the other hand, patients with agenesis of the ICA who suffer an ischemic insult are often asymptomatic because the collateral pathways support cerebral perfusion. ${ }^{5)}$ Collateral flow is most commonly supplied through the circle of the Willis, but rarely by embryonic vessels or the external carotid artery. Lie described six pathways of collateral circulation in individuals with agenesis of the ICA. ${ }^{10)}$ In type A, the unilateral absence of the ICA is associated with collateral circulation to the ipsilateral ACA through a patent AcomA and to the ipsilateral MCA from the posterior circulation through a hypertrophied posterior communicating artery (PcomA). In type B, the ipsilateral ACA and MCA are supplied across a patent AcomA. Type $\mathrm{C}$ reflects bilateral agenesis of the ICA; the anterior circulation is supplied via a carotid-vertebrobasilar anastomosis, and may involve hypertrophy of the PcomA. In type D there is unilateral agenesis of the cervical portions of the ICA and an intercavernous communication to the ipsilateral carotid siphon from the contralateral cavernous ICA. In type E, diminutive
ACAs are supplied by bilateral hypoplastic ICAs, and the MCAs are supplied by enlarged PcomAs. The type F pathway provides collateral flow to the distal ICA via transcranial anastomoses from the internal maxillary branches of the ECA system, the so-called rete mirabile. Our patient had no past history of ischemic attacks because there was sufficient cerebral perfusion (Lie's type B).

Patients with RAA and an isolated left innominate artery may manifest the subclavian steal syndrome because the left carotid artery and left subclavian arteries, which are isolated from the right aortic arch, are supplied retrogradely from the vertebrobasilar circulation. Our patient did not present with symptoms of the subclavian steal syndrome.

We reported a patient with an extremely rare, complex congenital anomaly of the aortic arch and cerebral arteries who presented with a ruptured aneurysm. It is important to understand the variety, vasculogenesis, and clinical problems associated with congenital blood vessel malformations.

\section{Conflicts of Interest Disclosure}

The authors report no conflict of interest concerning the materials or methods used in this study or the findings specified therein. All authors who are members of The Japanese Neurosurgical Society (JNS) have registered online Selfreported COI Disclosure Statements through the website for JNS members.

\section{References}

1) Stewart JR, Kincaid OW, Titus JL: Right aortic arch: plain film diagnosis and significance. Am J Roentgenol Radium Ther Nucl Med 97: 377-389, 1966

2) Pauliukas PA: The right aortic arch with mirror-image branching of brachiocephalic arteries and aplasia of the left brachiocephalic trunk: surgically cured rare cause of cerebrovascular insufficiency. Angiol Sosud Khir 11: 131-134, 2005

3) Singh B, Satyapal KS, Moodley J, Rajaruthnam P: Right aortic arch with isolated left brachiocephalic artery. Clin Anat 14: 47-51, 2001

4) Mart CR, Zachary CH, Kupferschmid JP, Weber HS: Tetralogy of Fallot with right aortic arch and isolation of the left innominate artery from a left-sided patent ductus arteriosus. Pediatr Cardiol 22: 58-59, 2001

5) Given CA 2nd, Huang-Hellinger F, Baker MD, Chepuri NB, Morris PP: Congenital absence of the internal carotid artery: case reports and review of the collateral circulation. AJNR Am J Neuroradiol 22: 19531959, 2001

6) Nakahira A, Hirai H, Sasaki Y, Suehiro S: Agenesis of the left carotid artery in the right aortic arch with Kommerell diverticulum. J Thorac Cardiovasc Surg 140: e5-e7, 2010

7) Afifi AK, Godersky JC, Menezes A, Smoker WR, Bell WE, Jacoby CG: Cerebral hemiatrophy, hypoplasia of internal carotid artery, and intracranial aneurysm. A rare association occurring in an infant. Arch Neurol 44: 232-235, 1987

8) Quint DJ, Boulos RS, Spera TD: Congenital absence of the cervical and petrous internal carotid artery with intercavernous anastomosis. AJNR Am J Neuroradiol 10: 435-439, 1989

9) Paşaoğlu L, Vural M, Ziraman I, Uyanik SA: Left internal carotid artery agenesis associated with basilar and left vertebral artery aneurysm. J Clin Imaging Sci 1: 60, 2011

10) Lie TA: Congenital Anomalies of the Carotid Arteries. Amsterdam, Excerpta Medica, 1968, pp 35-51

Corresponding author:

Kouji Yamasaki, MD, Department of Neurosurgery, Faculty of Medicine, University of Miyazaki, 5200 Kihara, Kiyotake, Miyazaki, Miyazaki 889-1692, Japan.

$\triangle$ kouji_yamasaki@med.miyazaki-u.ac.jp 